LA-UR-02-5486

Approved for public release; distribution is unlimited.

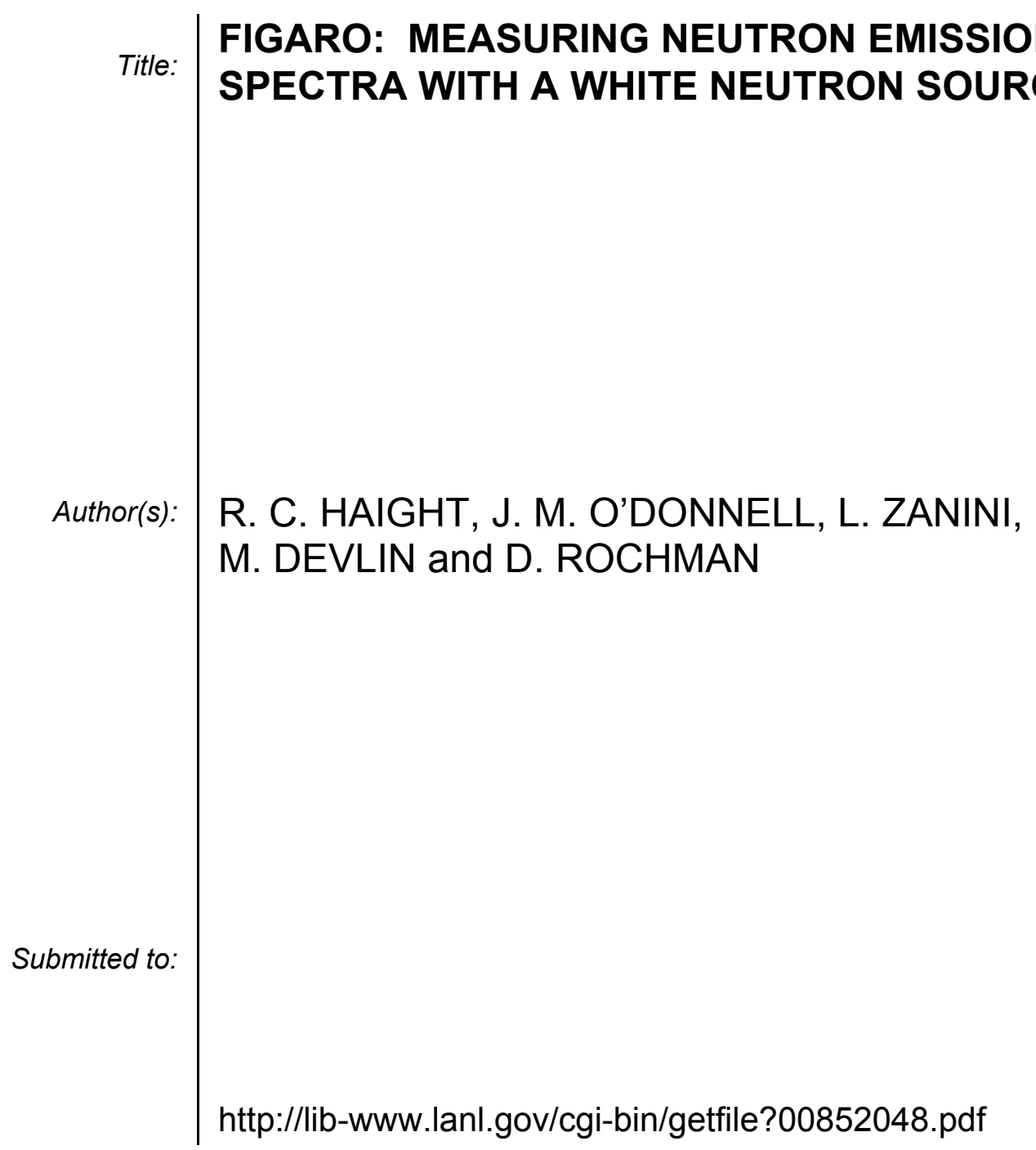

Los Alamos National Laboratory, an affirmative action/equal opportunity employer, is operated by the University of California for the U.S. Department of Energy under contract W-7405-ENG-36. By acceptance of this article, the publisher recognizes that the U.S. Government retains a nonexclusive, royaltyfree license to publish or reproduce the published form of this contribution, or to allow others to do so, for U.S. Government purposes. Los Alamos National Laboratory requests that the publisher identify this article as work performed under the auspices of the U.S. Department of Energy. Los Alamos National Laboratory strongly supports academic freedom and a researcher's right to publish; as an institution, however, the Laboratory does not endorse the viewpoint of a publication or guarantee its technical correctness. 


\title{
FIGARO: MEASURING NEUTRON EMISSION SPECTRA WITH A WHITE NEUTRON SOURCE
}

\author{
R. C. HAIGHT, J. M. O'DONNELL, L. ZANINI, \\ M. DEVLIN AND D. ROCHMAN \\ Los Alamos National Laboratory, \\ Los Alamos, NM 87545, USA \\ E-mail: haight@lanl.gov
}

\begin{abstract}
Neutron emission spectra from reactions induced by fast neutrons are of importance in basic physics and applications. Very few data are available in the literature over a wide range of incident neutron energies such as produced with a white neutron source. The FIGARO facility at the WNR/LANSCE neutron source has been established to measure such neutron emission over a range of incident neutron energies from 1 to over $100 \mathrm{MeV}$. Using the time-of-flight technique twice (once to determine the incident neutron energy, and again to determine the outgoing neutron energy), we are measuring neutron emission spectra for several reactions such as $\left(\mathbf{n}, \mathbf{n}^{\prime}\right)$ and $(\mathbf{n}, \mathbf{f})$. Neutron emission from inelastic scattering gives information on the level density of excited states of the target nucleus. Our first measurements are on structural materials such as iron.
\end{abstract}

\section{Introduction}

Neutron emission from reactions induced by fast neutrons has been a subject of much interest for many years due to the fundamental nature of the processes, free from the complications of the Coulomb interaction, and due to the many applications in nuclear energy, accelerator shielding, medical therapy, geological studies, and so forth. At the Los Alamos Neutron Science Center, we are developing capabilities for carrying out these measurements with a pulsed spallation, "white" neutron source. This is a report on the present status of our developmental activities.

Most measurements in the past have been conducted with pulsed, monoenergetic neutron sources where the sample to be investigated is near the source, and the energies and angular distributions of the emitted neutrons are determined by neutron detectors several meters from the sample. The time-of-flight technique is used to infer the emitted neutron energy. With a pulsed, "white" neutron source, in contrast, the incident neutron energy must be determined by time of flight between the source and the 
sample. The outgoing neutron energy is also deduced by time of flight from the sample to the detector. Thus, this approach is a double time-of-flight experiment where some signal needs to be detected at the sample to indicate (1) the time required for the neutron to travel from the source to the sample and (2) the beginning time for the emitted neutron before it travels toward the detector. For several reasons, this approach is difficult: The sample is several meters or more from the source and this results in a lower incident neutron flux; the repetition rate in accelerators producing the "white" source is usually lower than that at monoenergetic sources; the signal at the sample is difficult to obtain except for specialized samples that are themselves detectors such as scintillating materials or fission chambers containing actinides; the time-of-flight technique to infer the neutron energy requires a flight path between the sample and the detector with consequently small solid angles for detection, and, finally, neutrons are more difficult to detect than charged particles or photons.

In view of these difficulties, one might ask about the reasons for trying to measure neutron emission spectra with white neutron sources. There are several. First, with a white neutron source, measurements over the wide energy range are made naturally all at once in the same experiment. Thus questions of detector efficiency and how it might change from run-to-run are markedly reduced. Second, the collimation of a white neutron source can be much better than that of a monoenergetic source because, in the former, the sample is by necessity located much farther from the source to allow time-of-flight determination of the incident neutron energy. This added distance makes possible well-engineered shielding and collimation. Third, white neutron sources span a large energy range with no gaps. Our interests are for incident neutron energies from 1 to as high as perhaps 100 $\mathrm{MeV}$. Monoenergetic neutron sources have the well-known gap between 8 and $13 \mathrm{MeV}$ and they are not monoenergetic above about $17 \mathrm{MeV}$. Recent progress with quasi-monoenergetic sources have allowed neutron emission measurements in the gap region and above $17 \mathrm{MeV}$, but difficulties remain especially in the detection of lower energy neutrons, say below $3 \mathrm{MeV}$. Fourth, for some regions of monoenergetic neutron energies, triton beams or tritium targets are required, and these are much harder to find these days because of restrictions on handling tritium. Fifth, intense white neutron sources are now available from spallation reactions at several facilities whereas many monoenergetic neutron facilities have been closed.

If measurements of neutron emission can be made well with white neutron sources, then several areas of research can be addressed. Fission neutron spectra are well measured for selected incident neutron energies, but 
they are not well known for many energy regions and especially for incident neutron energies above $15 \mathrm{MeV}$. Detailed information can be obtained on how the spectrum changes as the incident neutron energy increases and higher order fission channels open up(second chance, third chance, etc.).

Nuclear level densities can be inferred from statistical neutron emission spectra from(n,n') interactions, but there is often the question of how much direct reactions contribute. With experimental data for a fixed channel (reaction type and Q-value) over a range of incident neutron energies and therefore a range of outgoing neutron energies, the direct and precompound reaction contributions should be identifiable as they increase with increasing neutron energy. Finally, with the detection of de-excitation gamma rays as triggers to the neutron emission, information will be obtained on the importance of angular momentum in the statistical neutron emission, in the level density in the residual nucleus, and in the following gamma-ray cascade in the decay of these unresolved, excited states. One goal of these measurements is to study possible structure in the nuclear level density as suggested by level counting (Fig. 1) and by modern theoretical work that uses the combinatorial approach or the Monte Carlo Shell Model.

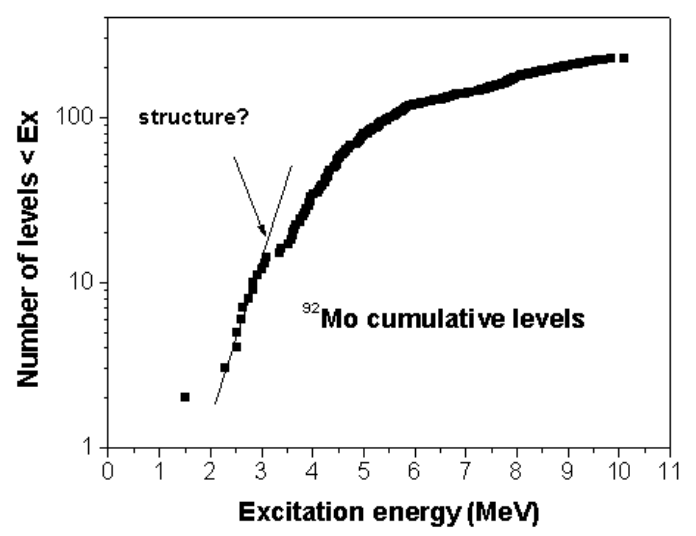

Figure 1. Number of known levels in ${ }^{92}$ Mo below excitation energy Ex. The data are from the ENSDF data base. The deviation of the level density from the exponential line around $3 \mathrm{MeV}$ either indicates missing levels or structure in the level density.

In addition to the basic physics interest in these processes, neutron emission spectra are of great importance in neutron transport, nuclear energy, transmutation of radioactive waste, and advanced accelerator applications. 


\section{Approach}

The present approach is to use the pulsed spallation source of fast neutrons at the Los Alamos Neutron Science Center (LANSCE); collimate the neutron beam to an experimental station where the neutrons interact with the sample material of interest; tag the interaction by emitted gamma rays, which also indicate the time of flight of the incident neutrons from the source and therefore their energy; and then detect the emitted neutrons with an array of detectors so that their energies can be determined by a second time of flight, that is from the sample to these neutron detectors. Schematically the approach is shown in Fig. 2.

The LANSCE spallation source of fast neutrons has been described previously. ${ }^{1}$ It is now operated with a time-averaged current of 5 microAmperes and produces about 35000 pulses per second with a typical spacing of 1.8 microseconds. The neutron spectrum can be found on the LANSCE Web site ${ }^{2}$ and is similar to a Maxwellian with a temperature of about 2 $\mathrm{MeV}$ with a high energy tail. We are using the 30-degree-Right flight path at a distance of 22 meters from the source. The beam is collimated to spot size of $1.3 \mathrm{~cm}$ diameter at the sample position.

Gamma-ray and neutron detectors view the sample as shown in Fig. 2. The facility is called FIGARO and has been described previously for experiments using only gamma-ray detectors. ${ }^{3}$ Typically the gamma-ray detectors are $15 \mathrm{~cm}$ from the sample on one side of the beam and the neutron detectors are located about 1 meter from the sample on the other side of the beam. In this developmental stage, we are using both HPGe detectors and $\mathrm{BaF}_{2}$ detectors. The germanium detector has the advantage of excellent resolution, but its efficiency and time resolution are only moderate. For the coaxial Ge-detector rated at $20 \%$ relative efficiency, the time resolution is at best $5 \mathrm{~ns}$ for a small dynamic range of gamma-ray energies in the $500-1500 \mathrm{keV}$ energy range. The $\mathrm{BaF}_{2}$ detector, on the other hand, has excellent timing characteristics together with good efficiency, but of course its energy resolution is much worse, being close to $10 \%$ in this energy region. The size of the $\mathrm{BaF}_{2}$ detector is $15 \mathrm{~cm}$ long with a $\mathrm{B}$-shape as described elsewhere. ${ }^{4}$

Liquid scintillators are used to detect the emitted neutrons. Because most of our interest is in neutron energies below $10 \mathrm{MeV}$, we use detectors that are $12.5 \mathrm{~cm}$ in diameter and only $5 \mathrm{~cm}$ thick. The scintillation material is EJ $301^{5}$, and it is viewed by a Hamamatsu R1250 $12.5 \mathrm{~cm}$ photomultiplier tube.

The electronics used are commercial modules. To digitize the neutron 


\section{Figaro}

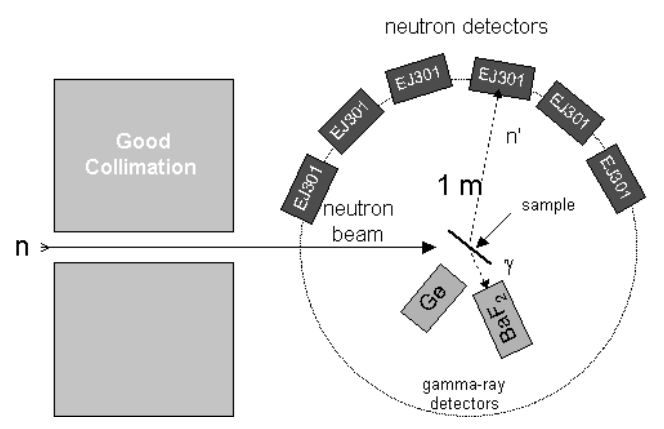

Figure 2. Schematic layout of FIGARO in it present configuration.

signals, we use FERA analog-to-digital converters with gates on the shorttime and long-time scintillation light components for pulse shape discrimination. These data, together with gamma-ray data from higher resolution digitizers, are stored in memory in the CAMAC crate and readout between macropulses of the neutron source. A typical PSD spectrum showing the differentiation between gamma-ray and neutron-induced events is shown in Fig. 3.

Data are acquired, analyzed and displayed by a PC-based system using the MIDAS $^{6}$ and ROOT $^{7}$ software packages running under the Linux operating system.

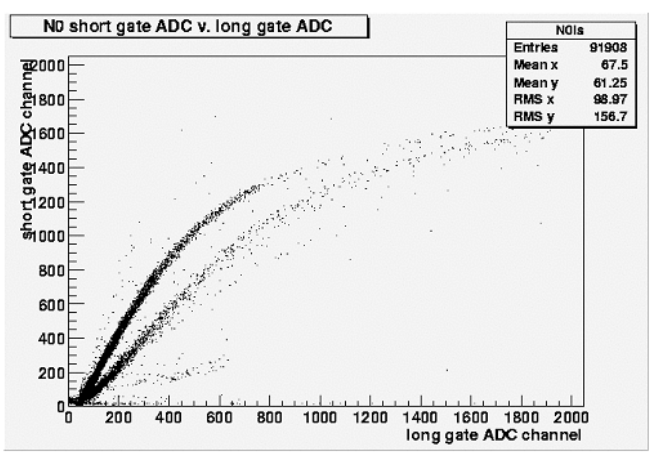

Figure 3. Typical pulse shape discrimination spectrum from EJ301 detectors. 


\section{Initial Experience}

The first experiments with the neutron detectors were fission neutron spectra as a function of incident neutron energy up to at least $100 \mathrm{MeV}{ }^{8}$ These studies, made with a fission chamber in the sample position, are providing useful data, which will be reported elsewhere. They also showed that the pulse-shape discrimination of the neutron detectors worked well. Because the efficiency for detecting fission is close to $100 \%$, the data have good statistics.

We are beginning initial measurements on gamma-neutron coincidences with iron, for which the dominant isotope is ${ }^{56} \mathrm{Fe}$. Inelastic scattering on ${ }^{56} \mathrm{Fe}$ leads to excited states in this nucleus that decay by gamma-ray cascades, and usually the cascade proceeds through the lowest $2^{+}$state at $847 \mathrm{keV}$. This line is very strong as observed by the HPGe detector. Even with the poorer resolution of the $\mathrm{BaF}_{2}$ detector, this line clearly stands out. We have concentrated on analyzing data taken with the $\mathrm{BaF}_{2}$ detector, for which the statistics are better.

Time-of-flight spectra are taken between the gamma-ray detectors and the neutron detectors. A typical example of such spectra is shown in Fig. 4. These data were selected for neutron energies between 1 and $10 \mathrm{MeV}$, for the $\mathrm{BaF}_{2}$ gamma-ray detector, and for the neutron detector at 90 degrees. By putting a cut on the PSD data, most of the gamma peak goes away and the time-independent background is significantly reduced. Some gamma

rays are still present because the separation between neutrons and gamma rays vanishes for small pulse heights.

\section{Issues}

Several issues have been identified as being important to this approach. They include time resolution, cross-talk between neutron and gamma-ray detectors, background reduction, counting rate, and detection efficiency.

Time resolution is clearly an issue for the short flight paths of 1 meter. For a resolution of $1 \mathrm{~ns}$, an energy resolution of $0.89 \mathrm{MeV}(8.9 \%)$ results at $10 \mathrm{MeV}$ emitted neutron energy or $0.028 \mathrm{MeV}(2.8 \%)$ at $1 \mathrm{MeV}$ emitted energy for thin detectors. The energy resolution scales for different time resolution, and thus the HPGe detector will lead to rather poor time resolution of the emitted neutrons. But at low energies of emitted neutrons, such as near $1 \mathrm{MeV}$, the neutron energy resolution will be dominated by the thickness of the neutron detector, even considering the modest time resolution of the HPGe gamma-ray detector. For our $5 \mathrm{~cm}$ thick detectors at 1 meter, the difference in possible flight path lengths inherently gives an 


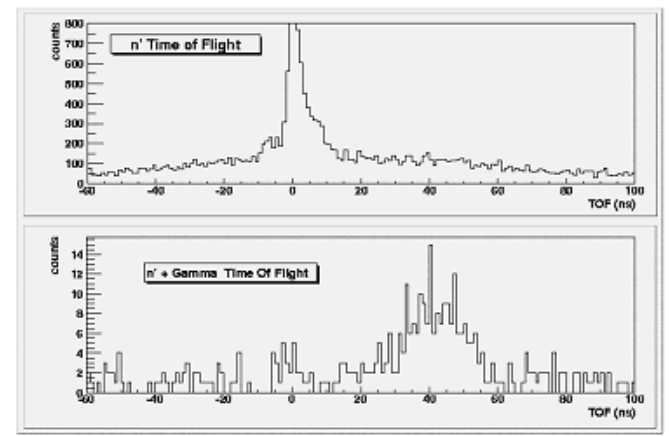

Figure 4. Time difference between events in the BaF2 gamma-ray detector and one of the neutron detectors. The top histogram includes all events; the lower histogram is gated on PSD for neutrons and also for incident neutron energies from 1.5 to $20 \mathrm{MeV}$ to eliminate complex events induced by faster neutrons. The emitted neutron spectrum, modified by the detector efficiency and the energy-to-time-of-flight conversion is clear from the lower histogram.

energy width of $10 \%$ for the emitted neutrons.

Details of the time resolution also need to be understood as indicated in Fig. 4. Not only is the FWHM of the gamma-gamma coincidence peak important, but the tails of this peak can determine how well the higher energy neutrons can be separated. One needs to understand how much of the gamma-gamma peak is underneath the faster neutrons. These details can in principle be determined experimentally with a gamma-ray source that emits coincident gamma rays. Consideration should be given also to the spectrum of this calibration source and how it compares with gamma rays expected in the experiment. The use of different thresholds for high and lower energy neutrons can help improve the separation between gamma rays and neutrons.

Cross talk is important between the gamma-ray detectors and the neutron detectors. Several types of cross talk can be imagined for events where gamma rays and neutrons come simply from the sample. Here we write $(G)$ for gamma-ray detector and $(\mathrm{N})$ for neutron detector:

1. Gamma ray detected by G; Neutron detected by $\mathrm{N}$

2. Gamma ray detected by G; Gamma ray detected by N

3. Neutron detected by G; Gamma ray detected by $\mathrm{N}$

4. Neutron detected by G; Neutron detected by $\mathrm{N}$

The desired events are (1) above. Events of type (2) are seen as the 
peak in Fig. 4 and are therefore necessary to determine the "t-zero" of the emitted neutrons. Events of type (3) appear as "faster-than-light" events and therefore can be eliminated. Type (4) events give time data that are in the range of data of type(1). They will not in general give counts that interfere with the peaks in the HPGe data, but they still should be watched.

To measure neutron emission spectra as a function of incident neutron energy, it will be important to have sufficient statistics so that reasonably small energy bins of incident and emitted neutron energies can be selected. This implies that the efficiency of detection for both gamma rays and neutrons should be increased. For neutrons, increase in efficiency is obtained by increasing the number of neutron detectors. For gamma rays, larger volume HPGe or other detectors are necessary.

\section{Future development}

This small array of detectors is being enhanced by adding more neutron detectors and a few more gamma-ray detectors. Up to 49 neutron detectors will eventually be installed in modules of 7 detectors each. The plan for one such module is shown in Fig. $5 .^{9}$ With these additional detectors, the efficiency for neutron detection will be increased by nearly an order of magnitude. Further, if better energy resolution is required, the detectors can be moved farther from sample and the gamma ray detectors and the resolution will improve proportionately, at a cost of reducing the count rate.

With the good resolution of HPGe detectors, elemental samples can often be used, at least for neutron energies below $(n, 2 n)$ thresholds. The specific isotope that is excited by inelastic scattering can be identified by its characteristic gamma rays. For elements with several abundant isotopes, data can be obtained for several isotopes all at the same time in one experiment. Because samples of at least several grams are required for these measurements, as for any neutron-emission measurement, the use of elemental samples will avoid the large cost of buying or borrowing isotopically enriched material.

\section{Acknowledgment}

This work has benefited from the use of the Los Alamos Neutron Science Center at the Los Alamos National Laboratory. This facility is funded by the US Department of Energy and operated by the University of California under Contract W-7405-ENG-36. 


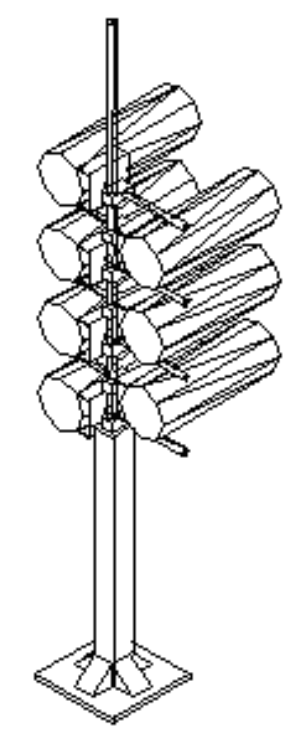

Figure 5. Design of a module of 7 neutron detectors as part of a large array?

\section{References}

1. P. W. Lisowski, C. D. Bowman, G. J. Russell, and S. A. Wender,it Nucl. Sci. Eng. 106, 208 (1990).

2. LANSCE Web site: http://www.lansce.lanl.gov

3. L. Zanini, A. Aprahamian, M. B. Chadwick, M. Devlin, R. C. Haight, J. X. Saladin, and P. G. Young, "Study of reactions of fast neutrons with nuclei with FIGARO at LANSCE," CAARI 2000: Sixteenth International Conference on the Application of Accelerators in Research and Industry, Denton, Texas (USA), November 1-5, 2000, ed. J. L Duggan and I. L. Morgan, AIP Conference Proceedings, Volume 576, pp. 346-349.

4. R. Reifarth, M. Heil, F. Kaeppeler, F. Voss, K. Wisshak, R. C. Haight, M. R. Dragowsky, M. M. Fowler, R. S. Rundberg, J. L. Ullmann, J. B. Wilhelmy, and E. H. Seabury, "New GEANT Simulations of Neutron Capture Experiments with a 4-pi BaF 2 Detector," Los Alamos Report LA-UR-01-4185 (July, 2001).

5. C. Hurlbut, Eljen Technology, Sweetwater, Texas, private communication (2002).

6. The MIDAS DAQ system, Web addresses http://midas.psi.ch/ and http://midas.triumf.ca/docmidas/

7. Rene Brun, Fons Rademakers, ROOT, An Object-Oriented Data Analysis Framework, Web address http://root.cern.ch/

8. T. Ethvignot et al., private communication (2001).

9. E. P. Chamberlin, Chamberlin Enterprises, Los Alamos, NM, USA, private communication (2002). 